\title{
Efecto de la intensidad de corte y actividad fotosintética en el crecimiento de grama (Paspalum notatum Flüggé) en el trópico seco centroamericano (Mesas de Moropotente, Nicaragua)
}

\author{
Karen Elizabeth Velásquez Meza ${ }^{1}$ \\ Jordi Bartolomé Fillelab ${ }^{2}$ \\ Kenny López Benavideza ${ }^{3}$
}

\begin{abstract}
RESUMEN
El trópico seco Centroamericano es un hábitat que está en peligro, y parte de él son las pasturas naturalizadas que cubren la mayor parte de las áreas, representadas en un 46\% del total (18.4 millones de hectáreas), siendo uno de los usos de la tierra más importantes (Kaimowitz D.1996) de pastoreo y son la base de la producción bovina en el ámbito regional. El objetivo de este trabajo es determinar el efecto de diferentes intensidades de corte y la actividad fotosintética, en el crecimiento del Paspalum notatum Flüggé. Teniendo como resultados que la intensidad de corte que produce mayor crecimiento del Paspalum notatum es la intensidad media, mostrando así que la frecuencia e intensidad de corte y la radiación fotosintética, se ve reflejado en el crecimiento del mismo.
\end{abstract}

Palabras claves: Trópico seco, Paspalum notatum, Intensidades de corte, radiación fotosintética.

1 UNAN-Managua/FAREM-Estelí Correo Electrónico: meza0788@yahoo.es

2 Universidad Autónoma de Barcelona España. Facultad de Veterinaria

3 UNAN-Managua/FAREM-Estelí, Estación Experimental para el Estudio del Trópico Seco “El Limón” 


\title{
Effect of cutting intensity and photosynthetic activity in the growth of grass (Paspalum notatum Flugge) in the dry tropics Central (Mesas de Moropotente, Nicaragua)
}

\author{
Karen Elizabeth Velásquez Meza ${ }^{1}$ \\ Jordi Bartolomé Fillelab ${ }^{2}$ \\ Kenny López Benavideza ${ }^{3}$
}

\begin{abstract}
The central American dry tropics habitat is in danger, and part of it are the natural grass that covers the mayor parts of the area, represented in a total of a $46 \%$ (18.4 million of hectares) being one of the world's most important (Kaimowitz D. 1996) pasturage and are the base of the bovine production in the region. The Objective of this job is to determine the effect of the different intensities of the cutting and the photosynthetic activities, in the growth of the Paspalum Natatum. Getting as a result that the intensity of the cut that provides the mayor growth of the Paspalum Natutum is the medium intensity, showing the frequency and intensity of the cutting and the photosynthetic radiation, it is reflected in the growth on itself.
\end{abstract}

Keywords: Tropics dry, Paspalum notatum, Cutting intensities, photosynthetic radiation.

1 UNAN-Managua/FAREM-Estelí E-mail: meza0788@yahoo.es 


\section{INTRODUCCIÓN}

El trópico seco, es el hábitat que está en mayor peligro en Centro América, actualmente reducido a menos del $1 \%$ de su magnitud original (Janzen, 1988). El desarrollo de las actividades agropecuarias, la causa principal del avance de la frontera agrícola, ha provocado la creación de pequeños parches o islotes de bosques dispersos en diferentes zonas, es decir, la fragmentación forestal. Mucha de la literatura acerca de ecosistemas tropicales y su buen manejo se refiere a bosques húmedos o sistemas de sabana (Saunder et al., 1991), mientras que en el trópico seco, han sido menos estudiados, pocos estudios examinan la interacción entre estos parches y su entorno, o viceversa (Janzen, 1988).

En América Central, el área en pasturas representa un $46 \%$ del total (18.4 millones de hectáreas), siendo uno de los usos de la tierra más importantes (Kaimowitz D.1996) y una de las principales actividades económicas para la generación de ingresos para subsistencia y empleo permanente para la población pobre (CATIE/ NORAD 2002).

En Nicaragua, el área de pasturas a finales del siglo XX ya habían alcanzado 4.2 millones de hectáreas, ocupadas con 2.65 millones de cabezas de ganado (Szott L. 2000), área que hacia el año 2005 mostró una ligera reducción (3.2 millones de hectáreas, con 3.5 millones de cabezas) (FAO 2008), posiblemente derivadas de la conversión de pasturas hacia charrales probablemente por la degradación de pasturas.

El crecimiento de la población es el factor más importante en el aumento de la demanda de productos agrícolas, ejerciendo a la vez presión sobre la expansión de las tierras de cultivo y el aprovechamiento de la madera como combustible, contribuyendo así a incrementar y acelerar la deforestación. Las presiones demográficas, aunque no son la causa principal del uso ineficiente de los recursos naturales y la degradación del medio ambiente, contribuyen a agravar la magnitud de los daños ecológicos por otras causas. Los sistemas deficientes de tenencia de la tierra, la falta de créditos, inadecuados precios de los productos agrícolas, políticas agropecuarias adversas, servicios deficientes de extensión agrícola y forestal, controles ineficientes e inestabilidad en el campo y la gestión inadecuada de los recursos contribuyen a potenciar los efectos negativos en el sector forestal (MARENA, 2001).

En la zona norcentral del país, se localiza el Paisaje Terrestre Protegido MiraflorMoropotente, con una extensión de 22,031.5 ha.

De acuerdo a Correa Do Carmo (2000), producto de la extensiva actividad pastoril y agrícola desarrollada por mucho tiempo, el área presenta un paisaje muy fragmentado, donde la actividad ganadera abarca un área considerable dentro de la reserva. Casasola (2000) estima que 5,584 ha son destinadas para el desarrollo de esta actividad en Moropotente, lo que ejerce una gran presión sobre los recursos naturales de las Mesas.

Otro estudio realizado por MARENA (2004), demuestra que en los últimos años parte de los cambios que han experimentado los pastizales en las Mesas de Moropotente, se deben a un manejo inadecuado que ha desplazado algunas especies de leguminosas que coexistían con el pasto natural, dando a lugar zonas con una fuerte proliferación de especies arvenses no palatables, que compiten con el pasto. Es importante, mencionar que la degradación del pasto influye directamente en su calidad nutricional y la productividad, lo que se expresa a través de las propiedades biofísicas del mismo, como biomasa, el índice de área foliar (LAI), y la altura (Numata et al; 2007).

Teniendo en cuenta lo antes mencionados, se planteó como objetivo de este estudio, determinar el efecto de diferentes intensidades de corte y la actividad fotosintética de la vegetación, en el crecimiento de 
la grama (Paspalum notatum Flüggé), situado en el trópico seco centroamericano (Mesas de Moropotente, Nicaragua).

\section{MÉTODOS}

Área de estudio. El paisaje terrestre protegido Miraflor-Moropotente, se encuentra ubicada en el Sector Norcentral de Nicaragua, en el extremo noreste del Municipio de Estelí, a unos $30 \mathrm{Km}$. de la cabecera departamental del mismo nombre, las coordenadas geográficas que ocupa el área son: $13^{\circ} 3$ ' $22^{\prime \prime}$ y $13^{\circ}$ 7'30" latitud Norte y 86 29' 15 " y $86^{\circ} 29$ ' 50" Longitud Oeste. Contiene partes planas como las Mesas de Moropotente a una altura de $1200 \mathrm{msnm}$.

El área protegida de Moropotente se ubica en los límites administrativos Municipales de Estelí por el Departamento de Estelí, y en La Concordia y San Sebastián de Yalí por el Departamento de Jinotega, de Nicaragua y tiene una superficie de 8534.9 ha, corresponde al 29\% del total del área protegida y se caracteriza por presentar suelos planos. Es una zona de aptitud ganadera, con características topográficas, fisiográficas muy especiales y tipos de suelo pedregosos; algunos suelos son pesados y pobres en nutrientes para agricultura. Dominan pastos naturales que no requieren riego.

Método. El método de muestreo empleado para la realización del estudios fue por conveniencia, ya que se trató de elegir aquellas parcelas que sean heterogéneas (similares en cuanto a orientación, sustrato y manejo), donde se establecieron parcelas de 20 × $20 \mathrm{~m}$ de exclusión en el que no puedan entrar los animales a pastar.

Por otra parte se destinaría a valorar el efecto de la intensidad de corte (como estimación de la intensidad de pastoreo) en el crecimiento de grama. Para ello se planteará un diseño de bloques al azar.
En cada parcela se establecerán 16 subparcelas $0.5 \times 0.5$ m. Cuatro tratamientos en bloques al azar, al finalizar la época seca e inicio de la época lluviosa: corte a ras de suelo o de intensidad alta (IA), corte a mitad de la altura o intensidad media (IM), corte a una cuarta parte de la altura o intensidad baja (IB) y sin corte o control (C).

Además se medirá la capacidad fotosintética o fAPAR, con el objetivo de medir la luz captada por el pasto y obtener una medida indirecta de la fotosíntesis que realiza la planta y por tanto, el crecimiento en biomasa de la misma. Se midió la luz interceptada por el pasto con un ceptómetro Accupar LP-80², que es un instrumento que capta la radiación visible, y es el rango espectral que utilizan las plantas para realizar la fotosíntesis.

Se realizaron 4 mediciones de luz con el ceptómetro Accupar LP-80, 1 sobre el pasto y 3 a ras de suelo. Primero se colocó el ceptómetro sobre el pasto, con la barra paralela al suelo. La medida de la parte superior de la barra de sensores recoge toda la luz que llega del Sol en el momento del muestreo (radiación incidente). La medida inferior es captada por un sensor adicional que apunta al suelo, éste capta la radiación solar que la planta refleja, es decir, la que no utiliza para fotosíntesis. En el caso de que haya mucho suelo descubierto, también se añade la cantidad de luz reflejada por el suelo.

Para determinar el efecto de diferentes intensidades de corte y actividad fotosintética de la vegetación, en el crecimiento de la grama, se utilizó la prueba no paramétrica de Kruskal Wallis (con $\mathrm{P}<0.05$ ).

1 El AccuPAR LP-80 es una barra 80 sensores formada por que son sensibles a la región visible del espectro, o PAR (radiación fotosintéticamente activa, en inglés), más un sensor adicional que se coloca en dirección coloca en dirección opuesta a la barra de sensor y un controlador que interpreta la señal PAR del sensor, lo que permite ver el valor PAR en una pantalla. También permite calcular el Índice de Área Foliar con cada medición PAR, considerando la radiación fotosintéticamente activa interceptada y disponible además de otras variables (Tecnología Omega). 
Para comparar los distintas intensidades de cortes en el crecimiento de la grama y se realizó un análisis de varianza simple y la prueba de comparación multiple de medias, con un nivel de confianza de un $95 \%$.

\section{RESULTADOS}

Efecto de diferentes intensidades de corte, en el crecimiento del paspalum notatum.

En las diferentes intensidades de corte, se encontraron diferencias significativas $(\mathrm{P}<0,05)$ entre ambas intensidades de cortes evaluadas, para las alturas encontradas por épocas $(\mathrm{P}<0,0001)$. Esto pudo estar influido por el decrecimiento de la época seca. (Cuadro 1)

El paspalum notatum mostró resultados significativo para cada una de las épocas, mostrando crecimiento en los meses de diciembre, enero y junio, decreciendo en los meses de marzo, abril y mayo, conforme a cada uno de las intensidades de corte establecidas (ver cuadro 1).

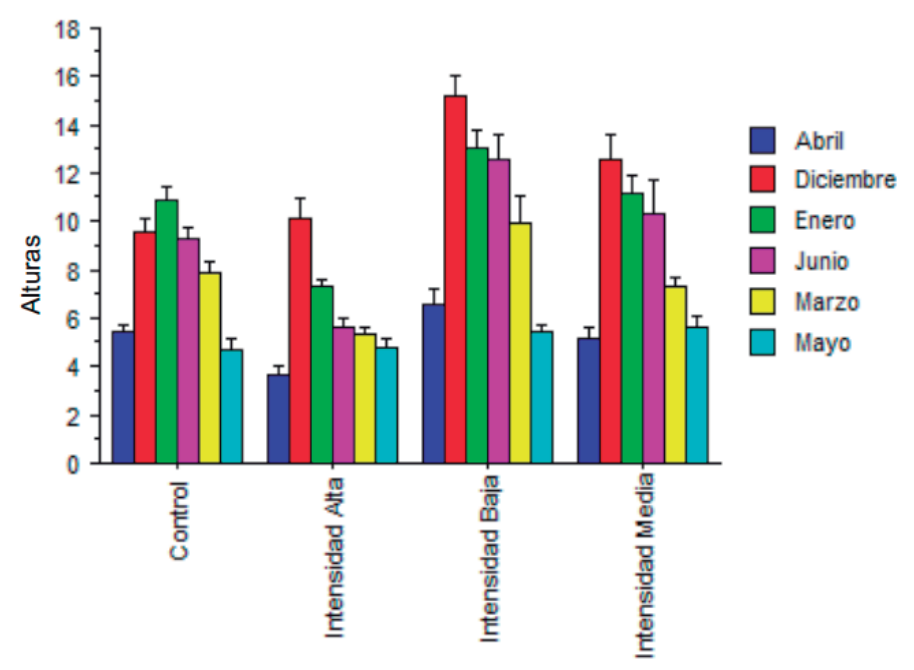

Figura 1. Crecimiento del Paspalum notatum en las diferentes intensidades de corte.
Paspalum notatum growth in different cutting intensities.

Cuadro 1: Efectos de las intensidades de corte en la alturas del Paspalum notatum. Effects intensities cutting heights in Paspalum notatum

\begin{tabular}{|c|c|c|c|c|c|}
\hline Tratamientos & Época & Ranks & Medias & D.E & $\mathrm{P}$ \\
\hline Control & Diciembre & $160,80 \mathrm{EFGH}$ & 9,60 & 1,71 & $<0,0001$ \\
\hline Control & Enero & $181,40 \mathrm{FGH}$ & 10,90 & 1,73 & \\
\hline Control & Marzo & $126,45 \mathrm{DEF}$ & 7,90 & 1,45 & \\
\hline Control & Abril & $60,50 \mathrm{ABC}$ & 5,40 & 1,07 & \\
\hline Control & Mayo & $43.45 \mathrm{AB}$ & 4,70 & 1,49 & \\
\hline Control & Junio & $157,05 \mathrm{EFG}$ & 9,30 & 1,34 & \\
\hline Int. Alta & Diciembre & $163,45 \mathrm{EFGH}$ & 10,10 & 2,81 & \\
\hline Int. Alta & Enero & $113,35 \mathrm{CDE}$ & 7,30 & 0,95 & \\
\hline Int. Alta & Marzo & $57,65 \mathrm{ABC}$ & 5,30 & 0,95 & \\
\hline Int. Alta & Abril & $19,85 \mathrm{AB}$ & 3,70 & 2,01 & \\
\hline Int. Alta & Mayo & $44,80 \mathrm{AB}$ & 4,80 & 1,03 & \\
\hline Int. Alta & Junio & 66,30 ABCD & 5,60 & 1,17 & \\
\hline Inte. Media & Diciembre & $196,50 \mathrm{GH}$ & 12,60 & 3,06 & \\
\hline Inte. Media & Enero & $183,70 \mathrm{FGH}$ & 11,20 & 2,25 & \\
\hline Inte. Media & Marzo & $156,35 \mathrm{EFG}$ & 7,30 & 1,16 & \\
\hline Inte. Media & Abril & $55,90 \mathrm{ABC}$ & 5,20 & 1,48 & \\
\hline Inte. Media & Mayo & $67,40 \mathrm{ABCD}$ & 5,60 & 1,51 & \\
\hline Inte. Media & Junio & $153,70 \mathrm{EFG}$ & 10,30 & 4,37 & \\
\hline Inte. Media & Diciembre & $221,00 \mathrm{H}$ & 15,20 & 2,70 & \\
\hline Inte. Media & Enero & $203,40 \mathrm{GH}$ & 13,00 & 2,49 & \\
\hline Inte. Media & Marzo & $156,35 \mathrm{EFG}$ & 9,90 & 3,60 & \\
\hline Inte. Media & Abril & 91,30 BCD & 5,20 & 2,01 & \\
\hline Inte. Media & Mayo & $60,50 \mathrm{ABC}$ & 5,40 & 1,07 & \\
\hline Inte. Media & Junio & $194,75 \mathrm{GH}$ & 12,60 & 3,24 & \\
\hline \multicolumn{6}{|c|}{ P nivel de significancia 95\%. P 95\% significance level. } \\
\hline \multicolumn{6}{|c|}{$\begin{array}{l}\text { Radiación fotosintética en el crecimiento del paspalum } \\
\text { notatum. }\end{array}$} \\
\hline \multicolumn{6}{|c|}{$\begin{array}{l}\text { De acuerdo a los análisis estadísticos realizados, los } \\
\text { datos no mostraron diferencia significativa alguna } \\
(\mathrm{P}>0,6496) \text {, con respecto a la variable altura }(\mathrm{P}< \\
0,0001) \text {. (Ver figura } 2) \text {. }\end{array}$} \\
\hline
\end{tabular}




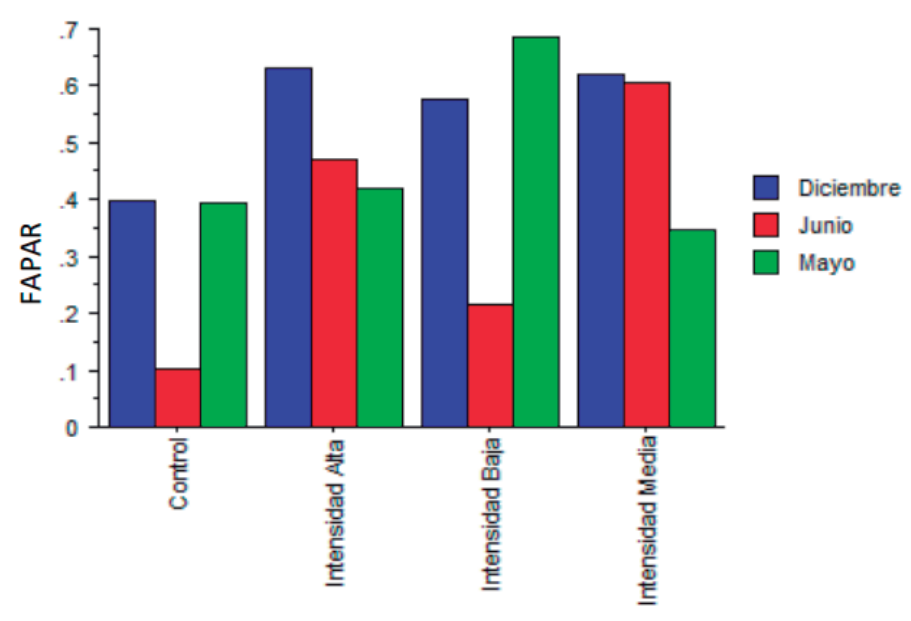

Figura 2. Variación de la FAPAR en las diferentes intensidades de corte en el paspalum notatum.

Cuadro 2. Radiación fotosintética en las intensidades de corte. Photosynthetic radiation intensities cut.

\begin{tabular}{llccc}
\hline \multicolumn{1}{c}{ Tratamientos } & \multicolumn{1}{c}{ Época } & Medias & D.E & \multicolumn{1}{c}{ P } \\
\hline Control & Diciembre & 0,40 & 0,11 & $<0,6496$ \\
Control & Mayo & 0.39 & 0,52 & \\
Control & Junio & 0,10 & 0,10 & \\
Int. Alta & Diciembre & 0.63 & 0,31 & \\
Int. Alta & Mayo & 0,42 & 0,11 & \\
Int. Alta & Junio & 0,47 & 0,43 & \\
Int. Media & Diciembre & 0.62 & 0,15 & \\
Int. Media & Mayo & 0,35 & 0,47 & \\
Int. Media & Junio & 0,60 & 0,04 & \\
Int. Baja & Diciembre & 0,57 & 0,36 & \\
Int. Baja & Mayo & 0,69 & 0,21 & \\
Int. Baja & Junio & 0,22 & 0,27 & \\
\hline
\end{tabular}

$P$ nivel de significancia 95\%. P 95\% significance level.

\section{DISCUSIÓN}

Efecto de diferentes intensidades de corte, en el crecimiento del paspalum notatum.

La altura del Paspalum notatum incremento en dependencia de las intensidades de corte $(\mathrm{p}>0.0001)$, mostrando un incremento en la altura en los meses de Diciembre, Enero y Junio, para el control de las muestras y para el resto de intensidades hay incremento solo en el mes de Diciembre y junio, el resto de meses las alturas decrecieron, por las intensidades del corte, factores climáticos y la salida de verano a invierno.

La frecuencia y la altura de corte afectaron el crecimiento de la atura del Paspalum notatum, en cada una de las intensidades, por lo tanto también hay un decrecimiento en la generación de biomasa fresca. Según Hodgkinson et al. (1989), se ha observado diferencias en las intensidades y frecuencias de corte de los pastos, siendo la intensidad media la que ha mostrado mayor incremento en la altura y cantidad de biomasa generada.

La alta intensidad de corte del Paspalum notatum aceleran la pérdida de cobertura del suelo. En este sentido, los cortes de la grama realizados a ras del suelo, afectan en forma significativa la disponibilidad de biomasa en más de un 50\%. De igual forma afectan el crecimiento de la misma. Los cortes realizados en la intensidad media afectan la disponibilidad de biomasa, aunque en menor proporción. Al respecto López et al. (2007) realizó una evaluación con intensidad de corte al en la alturas de Lolium perenne y Trifolium repens encontrado mayor producción de biomasa $(\mathrm{P} \leq 0,001)$ el corte de intensidad media.

Los cortes o pastoreos realizados en forma intensiva afectan la producción de nuevos brotes de las plantas forrajeras por dos aspectos, la primera es la eliminación de las reservas orgánicas o carbohidratos no estructurales localizados en los tallos y/o coronas y la segunda por falta de área foliar para el reinicio de la fotosíntesis después del pastoreo o corte del pasto (Rincón et al.2008).

\section{Radiación fotosintética en el crecimiento del Paspalum} notatum.

La radiación solar es la principal fuente de energía para los procesos fisiológicos que se llevan a cabo en 
las plantas. La fracción de radiación solar absorbida en el rango espectral comprendido entre 400-700 nm corresponde a la radiación utilizada en la fotosíntesis, por lo que se denomina radiación fotosintéticamente activa (PAR) y es aproximadamente el $45 \%$ de la radiación solar global. La radiación PAR es el factor medioambiental que determina principalmente el crecimiento de las plantas (Mariscal et al., 2000).

Los valores de la fracción de radiación fotosintéticamente activa absorbida (fAPAR) por el Paspalum notatum para la realización de fotosíntesis y en su crecimiento se representan en la figura 2, donde los valores más altos de absorción lumínica se muestran en la intensidad de corte alta y media, en las diferentes épocas de muestreo, reflejando que los meses en que el Paspalum notatum decrece por ser época de verano, hay mayor radiación de luz, pero no es utilizada por la planta, mientas que a la entrada del invierno, la especie trata de aprovechar mayor cantidad de luz solar para realizar la fotosíntesis y crecimiento en los diferentes intensidades de cortes, lo que se traduce en un aumento en altura y biomasa del mismo.

Según Nouvellon Y 2000, además de la radiación fotosintética, las especies necesitan de otros factores como precipitación, temperatura para alcanzar un mayor desarrollo y crecimiento y así lograr una mayor productividad.

Es importante también tener en cuenta que las plantas en general, utilizan sólo la luz visible como fuente de energía para la fijación de dióxido de carbono en la fotosíntesis que controla el desarrollo de las mismas.

Sin embargo el Paspalum notatum por ser una especie de ambiente cálido y seco utilizan una vía fotosintética diferente llamada $\mathrm{C} 4$, que es más resistente a la alta luminosidad y temperatura y es más eficiente en la tasa de fijación de carbono por molécula de agua perdida en la transpiración, por lo que los pastos en estas zonas tienen un crecimiento más rápido y resistente a las sequías según Mariscal 2000. Pero a pesar de tener un rápido crecimiento, los cortes de las diferentes intensidades, hace que el Paspalum notatum, no alcance alturas demasiado altas.

\section{CONCLUSIONES}

En base a los resultados obtenidos, la intensidad de corte que produce mayor crecimiento del Paspalum notatum fue la intensidad media, mostrando así que la frecuencia e intensidad de corte y la radiación fotosintética, se ve reflejado en el crecimiento del mismo y para asegurar el desarrollo y sustentabilidad de la actividad ganadera en las Mesas de Moropotente, se deberán impulsar alternativas de producción sostenible, donde el pastoreo sea de intensidad media, utilizando alternativas como: los sistemas silvopastoriles que optimice el uso de la tierra garantizando mayor productividad e ingresos y, pero conservando los recursos naturales.

\section{AGRADECIMIENTOS}

"Mi agradecimiento especial a la Facultad Regional Multidisciplinaria de Estelí (UNAN - MANAGUA / FAREM ESTELI) y al Proyecto Integral de Manejo de Cuencas Hidrográficas, Agua y Saneamiento (MARENA-PIMMCHAS), por su colaboración técnica y financiera, la cuál ha sido posible gracias a la cooperación de los Pueblos y Gobiernos de Canadá y Nicaragua.

\section{REFERENCIAS}

Casasola F. 2000. "Productividad de los sistemas silvopastoriles tradicionales en Moropotente, Estelí, Nicaragua". Tesis de maestría. Turrialba, Costa Rica.

CATIE/NORAD Proyecto "Desarrollo participativo de alternativas de uso sostenible de la tierra para pasturas degradadas en Centroamérica" Turrialba, 2002, $28 \mathrm{p}$ 
Correa Do C. 2000. "Evaluación de un paisaje fragmentado para la conservación y recuperación de biodiversidad área demostrativa Miraflor Moropotente, Estelí, Nicaragua". Turrialba (Costa Rica). 148 p.

FAO (Food and Agriculture Organization of the United Nations) 2008. FAOSTAT. (Consultado en 01-052008).URL: http://faostat.fao.org

Hodgkinson, C. K., M. Ludlow, J. Mott, and Z. Baruch. 1989. Comparative responses of the savanna grasses Cencrhus ciliaris and Themeda triandra to defoliation. Oecologia 79: 45-52.

Janzen, D.H. 1988. Management of habitat fragments in a tropical dry forest: Growth. Annals of the Missouri Botanical Garden 75: 105-106.

Kaimowitz D. Livestock and deforestation. Central America in the 1980s and 1990s: A Policy Perspective. CIFOR, Jakarta, Indonesia1996; 88 p López IF, Balocchio A, Corner J. 2007. Evaluación de la frecuencia e intensidad de pastoreo sobre atributos productivos y de sustentabilidad en producción de leche. Informe primer año, Valdivia, Chile: Instituto de Producción Animal; Instituto de Ingeniería Agraria y de suelos, Facultad de Ciencia Agrarias, universidad Austral de Chile.

MARENA. 2001. Informe del estado actual del medio ambiente, Managua, Nicaragua.

MARENA. III Informe Geo del Estado del Ambiente de Nicaragua 2003-2006.
Mariscal M.J. et al. 2000. Modelling and measurement of radiation interception by olive canopies. Agricultural and Forest Meteorology, 100:183197.

Nouvellon Y, Lo seen D, Rambal S, Bégué A, Moran MS, Kerr Y, Qi J. 2000. Time course of radiation use efficiency in a shortgrass ecosystem: consequences for remotely sensed estimation of primary production. Remote Sensing of Environment, 71:43-55.

Reyes. JJ. 2005. Efecto de la intensidad de pastoreo en el sistema suelo-planta-animal, en condiciones de bajos insumos. Instituto de ciencia animal, San José de Las Tejas, La Habana.

Rincón A, Ligarreto GA, Garay E. 2008. Producción de forraje en los pastos Brachiaria decumbenscv amargo y Brachiaria brizantha cv Toledo sometidos a tres frecuencias y a dos intensidades de defoliación en condiciones del Piedemonte llanero colombiano. Rev Facul Nal Agr medellín 61(1):4336-4346.

Sanuder, D. A., Hobbes, R. J. y Margules, C. R. 1991. Biological consequences of ecosystem fragmentation: a Review, Conservation Biology 5: 18-23.

Szott L, Ibrahim M, Beer J. The hamburger connection hangover: cattle pasture, land degradation and alternative land use in Central America. CATIE Serie Técnica 2000. 